\title{
Variable environments select for short lifespan
}

Martin I. Lind ${ }^{1, *}$, Irja I. Ratikainen ${ }^{2}$, Johan Andersson ${ }^{1}$, Hanne Carlsson ${ }^{1,3}$, Therese Krieg ${ }^{1}$, Tuuli Larva $^{1}$ \& Alexei A. Maklakov ${ }^{1,3}$

${ }^{1}$ Animal Ecology, Department of Ecology and Genetics, Uppsala University, 75236

Uppsala, Sweden

${ }^{2}$ Centre for Biodiversity Dynamics, Department of Biology, Norwegian University of Science and Technology (NTNU), Høgskoleringen 5, NO-7491 Trondheim, Norway

${ }^{3}$ School of Biological Sciences, University of East Anglia, Norwich Research Park, Norwich NR4 7TJ, UK

Martin I. Lind - martin.i.lind@gmail.com *Corresponding author 


\begin{abstract}
The role of environmental variation in the evolution of lifespan is contested. Classic theory suggests that variable environments result in evolution of long life but novel theoretical breakthroughs show that environmental variation can instead select for short lifespan when the changes are rapid relative to generation time. Here we combined simulation modelling and experimental evolution to study the evolution of lifespan in response to temperature variation to provide the first empirical test of the new theory. Genetically diverse populations of the outcrossing nematode Caenorhabditis remanei, were exposed to a novel, stressful temperature for 30 generations, in either stable, slowly increasing or fluctuating temperature regimes. We found evolution of shorter lifespan in populations evolving in rapidly fluctuating environments both in simulation models and in real populations supporting the new theory. While climate warming is predicted to increase environmental stochasticity, our results show that fast temperature cycles rapidly select for short lifespan.
\end{abstract}

Key words: ageing, environmental heterogeneity, experimental evolution, lifespan 


\section{Introduction}

Natural environments are seldom stable, but often change directionally or fluctuate at different timescales. Therefore, the role of environmental variation in the adaptive expression of phenotypes commands considerable interest, since it can influence evolutionary outcomes (Murphy 1968; Tuljapurkar and Orzack 1980; Charlesworth 1994; Roff 2002; Wilbur and Rudolf 2006; Morris et al. 2008; Cotto and Ronce 2014; Botero et al. 2015; Tufto 2015; Ratikainen and Kokko 2019).

Evolution in novel and variable environments can have far-reaching consequences for the evolution of life-history strategies, especially for the trade-off between growth, reproduction and somatic maintenance, which directly affects the evolution of lifespan. The theoretical models often make opposing predictions, from the evolution of robust and long-lived individuals, to the evolution of short lifespan in heterogeneous environments (Murphy 1968; Tuljapurkar and Orzack 1980; Charlesworth 1994; Wilbur and Rudolf 2006; Morris et al. 2008; Cotto and Ronce 2014; Ratikainen and Kokko 2019). Evolution of lifespan in novel but stable environments is generally considered as changes in age-specific mortality (Williams et al. 2006), where increased adult mortality is predicted to result in short lifespan, unless mortality is also condition dependent (Stearns et al. 2000; Reznick et al. 2004; Chen and Maklakov 2012). The first set of theories predict the evolution of longer lifespan in both new and variable environments, as long as the new environment is also stressful (Parsons 1995; Kenyon 2005). Empirical studies show that selection in stressful but stable environments often results in the evolution of stress resistance and longer lifespan but trade-offs with growth or reproduction (Sørensen et al. 2003; Bubliy and Loeschcke 2005; Chen et al. 2016; Lind et al. 2017). Traditionally, the 
same result has been predicted under environmental heterogeneity, since the organism should survive the unfavourable periods of stress to reproduce under more favourable conditions (Murphy 1968; Tuljapurkar and Orzack 1980; Charlesworth 1994; Wilbur and Rudolf 2006; Morris et al. 2008).

However, recent theoretical findings suggests instead that adaptation to constant and variable environments may select for either short or long lifespan, depending upon the time-scale of environmental variation relative to the generation time and the presence or absence of reversible plasticity (Cotto and Ronce 2014; Ratikainen and Kokko 2019). Central to the new theories is the concept of phenotypic mismatch when the environment changes. Phenotypic mismatch can select for the evolution of short lifespan in order to complete most of reproduction in the environment of development (Cotto and Ronce 2014; Ratikainen and Kokko 2019). Alternatively, phenotypic mismatch can select for the evolution of reversible plasticity to track environmental changes, accompanied by the evolution of long lifespan (Ratikainen and Kokko 2019). Without reversible plasticity, stable but novel as well as heterogeneous environments should select for short lifespan (Cotto and Ronce 2014). Since adaptation always proceeds with a lag, an adapting population is not instantly at the new fitness peak (Lande and Shannon 1996; Kopp and Matuszewski 2014). Because selection gradients on traits decline with age (Medawar 1952; Hamilton 1966; Caswell and Shyu 2017), older individuals have a weaker response to selection and are thus further from the new fitness peak. Therefore, in a population with age structure, adaptation to new environments is associated with reduced fitness, which is most severe for late age classes, resulting in accumulation of mutations with lateacting deleterious effects. While a rapid shift to a new stable environment only results 
in a transient effect, a population in a continuously moving or fluctuating environment will never be fully adapted, and selection against somatic maintenance strongest (Cotto and Ronce 2014).

Recently, the evolution of lifespan in heterogeneous environment was modelled in depth by Ratikainen and Kokko (2019). By considering that variable environments could result in the evolution of reversible plasticity, they demonstrated that the predictions depend upon the ability of the individual to adjust its phenotype to a changing environment along the life course. Therefore, the prediction by Cotto and Ronce (2014) only holds if reversible plasticity is not evolving. In contrast, the model by Ratikainen and Kokko (2019) predicts that if reversible plasticity is costly to express, and reproduction and lifespan are traded off against each other, fast changing environments will result in the evolution of long lifespan and lifelong reversible plasticity in order for the individual to track the changes in its environment. Less variable environments, however, select against costly reversible plasticity and instead select for increased investment in reproduction, which is traded off against lifespan (Ratikainen and Kokko 2019).

Finally, an environment that fluctuates between two states could also be seen as two time-constrained opportunities, where individuals are expected to reproduce quickly in the environment they developed in, thus trading off lifespan against early-life fitness. This scenario has never been formally modelled, to our knowledge, but should apply in cases when an individual is less likely to see the return of the developmental environment once the environment has changed. In support of this hypothesis, time constraints select for traits such as fast development and growth (Johansson and Rowe 
1999; Laugen et al. 2003; Lind et al. 2011) that are genetically correlated with short lifespan (Lee et al. 2013; Lind et al. 2017).

Taken together, these theories provide conflicting predictions as to how life-history evolution is affected by adaptation to new and heterogeneous environments. Given the predicted increase in both mean and variance of environmental factors during ongoing climate warming (Stocker 2014), understanding how fluctuating environments will affect life-history evolution is becoming increasingly relevant and paramount yet these predictions have not been tested empirically.

We set out to test these predictions using an experimental evolution approach in the dioecious nematode Caenorhabditis remanei, adapting to five different temperature regimes. Genetically heterogeneous replicate populations, previously adapted to $20^{\circ} \mathrm{C}$ ambient temperature, were evolving for 30 generations in i) control environment $\left(20^{\circ} \mathrm{C}\right)$; ii) novel stressful environment $\left(25^{\circ} \mathrm{C}\right)$; iii) slow warming to $25^{\circ} \mathrm{C}$; a heterogeneous novel environment with fluctuating temperatures between $20^{\circ} \mathrm{C}$ and $25^{\circ} \mathrm{C}$ either iv) fast, every $2^{\text {nd }}$ generation; or v) slow, every 10 th generation (Figure 1). Importantly, we used an overlapping generations setting, to test the model predictions. The experimental evolution was accompanied by extending the original model of Ratikainen and Kokko (2019) to deal with environments where an organism is not likely to see the return of the initial environment after an environmental shift. We found the evolution of short lifespan in environments cycling every $2^{\text {nd }}$ generation, both empirically and in the simulation results. 


\section{Methodology}

\section{Experimental evolution}

Experimental evolution was conducted with the SP8 strain of C. remanei as founder population (obtained from N. Timmermeyer, then at the Department of Biology,

University of Tübingen, Germany). This strain harbours substantial standing genetic variation for life-history traits and responds readily to selection (Chen and Maklakov 2012, 2014; Lind et al. 2017). The strain has been lab-adapted for 15 generations at $20^{\circ} \mathrm{C}$ prior to the setup of the experimental evolution.

\section{Experimental evolution regimes}

30 overlapping generations

Temperature-specific generation times

Six replicate populations per evolutionary regime

Control $20^{\circ} \mathrm{C}$

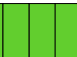

Warm $25^{\circ} \mathrm{C}$

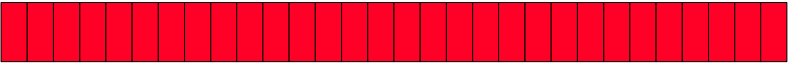

Fast temperature cycles

Increased warming

Slow temperature cycles

Generations

Figure 1. Overview of the experimental evolution regimes. Each of the five regimes consisted of six replicate populations. Generation time was 4 days in $20^{\circ} \mathrm{C}$ and 3.4 days in $25^{\circ} \mathrm{C}$, and we used an overlapping generations setting. 
The experimental evolution procedure is described in detail in Lind et al. (2019 bioRxiv) and outlined in figure 1 . We conducted experimental evolution for 30 generations using three different temperature settings: $20^{\circ} \mathrm{C}, 25^{\circ} \mathrm{C}$ and slow increase from $20^{\circ} \mathrm{C}$ to $25^{\circ} \mathrm{C}$ by $0.1^{\circ} \mathrm{C}$ every 2.13 days (rounded to whole days). We set up five selection regimes. Control $20^{\circ} \mathrm{C}$ populations were experiencing $20^{\circ} \mathrm{C}$ for 30 generations, and $\operatorname{Warm} 25^{\circ} \mathrm{C}$ populations were experiencing $25^{\circ} \mathrm{C}$ for 30 generations. Increased warming populations experienced slow increase from $20^{\circ} \mathrm{C}$ at the start of the experiment to $25^{\circ} \mathrm{C}$ on the last day of selection in slow incremental steps of $0.1{ }^{\circ} \mathrm{C}$ over the period of 30 generations. Slow temperature cycles was experiencing the first ten generations from the start of the experiment in $20^{\circ} \mathrm{C}$, then were switched for the next ten generations to $25^{\circ} \mathrm{C}$, and then switched back again for the remaining ten generations to $20^{\circ} \mathrm{C}$. Finally, the Fast temperature cycles regime spent the first two generations at the start of the experiment in $20^{\circ} \mathrm{C}$, then were switched for two generations to $25^{\circ} \mathrm{C}$ and this cycle was repeated 7.5 times (the last two generations were in $20^{\circ} \mathrm{C}$ ). Importantly, by generation we refer to generation time, as we used an overlapping generations setting.

We defined generation time as the average difference in age between parents and offspring (Charlesworth 1994) in $20^{\circ} \mathrm{C}$ and $25^{\circ} \mathrm{C}$, and it was calculated by solving the Euler-Lotka equation from the life-table of age-specific survival and reproduction (Stearns 1992; Charlesworth 1994) using trial data from the original SP8 line. Generation time was 4.0 days in $20^{\circ} \mathrm{C}$ and 3.4 days in $25^{\circ} \mathrm{C}$, which resulted in 120 days of selection for Control $20^{\circ} \mathrm{C}, 114$ days for Slow temperature cycles, 110 days for Increased warming and Fast temperature cycles and finally 101 days for Warm $25^{\circ} \mathrm{C}$. To ensure equal exposure to the two temperature treatments during biological time, the worms spent shorter chronological time in $25^{\circ} \mathrm{C}$ than in $20^{\circ} \mathrm{C}$ for the two 
temperature cycle treatments, because generation time is faster in $25^{\circ} \mathrm{C}$.

During experimental evolution, the populations were kept on $92 \mathrm{~mm}$ NGM-plates (Stiernagle 2006). The antibiotics kanamycin and streptomycin, and the fungicide nystatin was added to agar and LB to combat bacterial and fungal infections (Lionaki and Tavernarakis 2013; Lind et al. 2016). We seeded the plates with the antibiotic resistant E. coli strain OP50-1 (pUC4K) from J. Ewbank at Centre d'Immunologie de Marseille- Luminy, France. The lines were kept in exponential growth phase by cutting out a bit of agar containing 150 individuals of mixed ages and transferring this to freshly seeded plates, in order to keep the populations age-structured in overlapping generations. Transfer was conducted when needed (every one to two days) and plates were never allowed to run out of food. We set up six independent replicate populations of each selection treatment, resulting in 30 experimental populations in total. All populations were expanded in size for two generations and frozen after 10, 20 and 30 generations. For generations 10 and 20, a random subset of each population was used for freezing, to not interfere with the ongoing experiment.

\section{Lifespan assays}

Before assays, worms were recovered from freezing and grown for two generations in common garden at $20^{\circ} \mathrm{C}$, each generation synchronized by bleaching, a standard procedure that kills all life-stages but the eggs (Stiernagle 2006). Lifespan assays were established using ten age-synchronised females in the L4 stage as target worms, and to keep them mated they were kept together with ten background males from the original SP8 line. Worms were developing in the testing temperature since eggs. Worms were transferred daily, and sex ratio was kept at 1:1 by matching the number of background males to the number of testing females alive. The assay was run using 
four replicate plates of each line and temperature $\left(20^{\circ} \mathrm{C}\right.$ and $\left.25^{\circ} \mathrm{C}\right)$ combination, resulting in 240 plates (five selection regimes $\times$ six replicate lines $\times$ two temperatures $\times$ four replicate plates) and 2400 target worms. To separate the effect of temperature and cabinet, two cabinets were used for each temperature, and 2 replicate plates of each line was run in each cabinet. Worms were scored as dead if they did not respond to gentle prodding by the picker. Missing worms and females dying of matricide (bagging, internal hatching of eggs) were censored.

\section{Statistical analyses}

Survival was analysed using Cox proportional hazard models with Gaussian random effects implemented in the coxme package for $R$ 3.2.2. Selection treatment and testing temperature were fitted as fixed factors, and population, plate and testing cabinet (climate chamber) as random effects.

\section{Simulation model}

We modified the individual-based simulation model by Ratikainen and Kokko (2019) to investigate co-evolution of reversible plasticity and the trade-off between reproduction and lifespan in a two-state environment $E$ with different length cycles $l$ in the two different states. We operationally define the environmental state as the state of the phenotype that is optimal for this environment. An individual with the other phenotype has a mismatch $m_{i, t}$. The genotype of an individual has two loci, where the $u$ locus determines an individual's propensity to update its phenotype and $r$ its reproductive effort. Individual survival probability, $s$, is negatively affected by phenotypic mismatch $m_{i, t}$ with the environment, and trades-off with reproduction, $r$, so that $s_{i, t}=\alpha_{0}+m_{i, t}+\rho r^{2}$. In each time step, the individual either updates or does not 
update its phenotype, and we model the probability of updating as an increasing function of the time $T_{u}$ since the last update, and the parameter $u$ determines how steeply the probability rises with $T_{u}: P[\text { updating }]_{i}=1-e^{-u_{i} T_{u, i}}$. We assume developmental plasticity, and that all individuals perform an update at the time of birth. Updating reset the mismatch but has a small probability $\varepsilon$ of resetting to the wrong environment. This can be due to the inability of an organism to perfectly sense the state of the environment.

All individuals can reproduce in each time step, and brood size $c_{i, t}$ depends on the parent's investment in reproduction $r$, its mismatch $m$, and if it updated and therefore have to pay a cost for updating. $c_{i, t}=\min \left(10, r_{i}\right) e^{-m_{i, t}^{2}}\left(1-\kappa U_{i, t}\right)$ where $\kappa$ is the penalty for updating in the current timestep and $U$ takes the value 1 for individuals that updates and 0 for others. Offspring inherit the genotype of the parent, including $u$ and $r$, with some small probability of mutation. When an adult individual die, the vacancy in the population will be filled by a random recruit; juveniles that are not recruit are removed. We use a constant population of 2000 individuals over 20000 generations.

We vary the environmental cycle length, $l$, in steps from $l=1$ to $l=20$. For each $l$ we start the simulation from a range of genetic values for $u$ and $r$. To investigate the robustness of our results we also vary the background mortality $\alpha_{0}$ and the mismatch $m$ of an individual with the wrong phenotypic state for the environment. The $R$ code for the model along with a detailed description of the model can be found in supplementary materials II. 


\section{Results}

We found that female lifespan differed between the selection treatments $\left(\chi^{2}=865.3\right.$, $\mathrm{df}=4, \mathrm{p}<0.001)$, and that lifespan was shorter in $25^{\circ} \mathrm{C}\left(\chi^{2}=17.843, \mathrm{df}=1, \mathrm{p}<\right.$ $0.001)$, but no selection treatment $\times$ temperature interaction was detected $\left(\chi^{2}=1.44\right.$, $\mathrm{df}=4, \mathrm{p}=0.837$ ), implying that the ordering of the selection treatment was not different in the two temperatures. Extracting the individual coefficients from the minimal model (without interaction term) shows that the treatment effect is caused by a significantly longer lifespan of Warm $25^{\circ} \mathrm{C}$ and Increased warming compared to the short-lived Fast temperature cycles. Control $20^{\circ} \mathrm{C}$ and Slow temperature cycles had intermediate lifespan (Figure 2, Table 1, Supplementary figure 1).
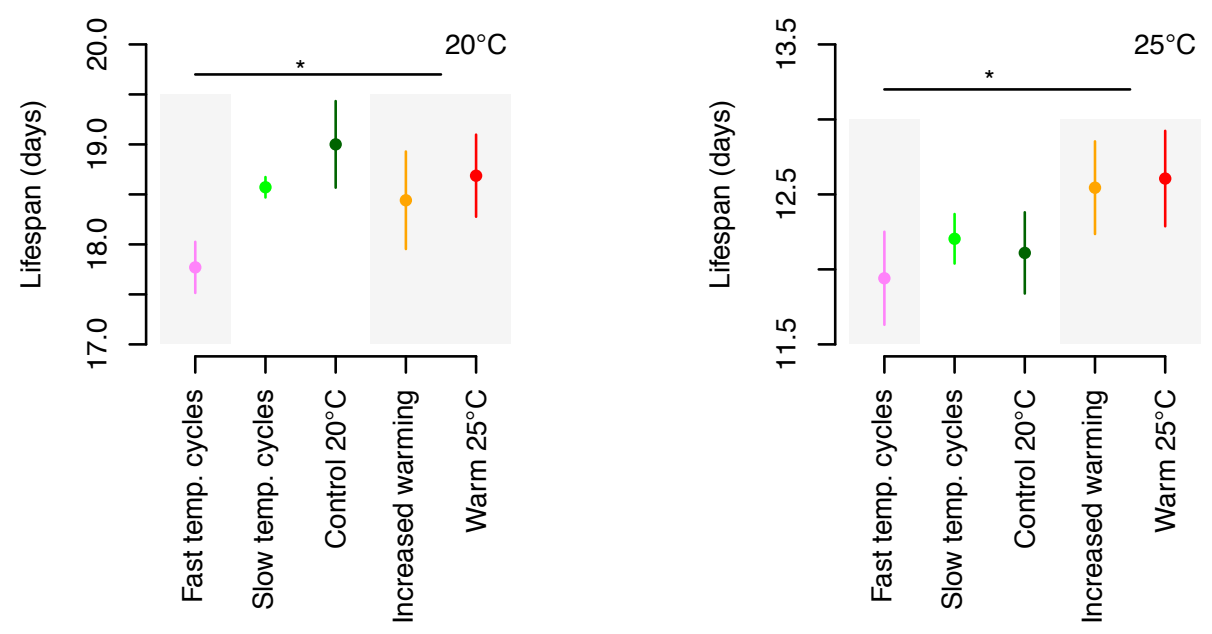

Figure 2. Mean lifespan in (A) $20^{\circ} \mathrm{C}$ and (B) $25^{\circ} \mathrm{C}$. Symbols represent experimental evolution regime (mean \pm SE based upon population means). The grey boxes represent the significant differences in lifespan across both temperatures, no temperature interaction was detected. 
Table 1. Coefficients from the best model of the survival analysis, which does not involve the nonsignificant interaction between Regime and Temperature. All comparisons in the table are made between the Fast cycle regime and the temperature treatment $20^{\circ} \mathrm{C}$.

\begin{tabular}{lcccrc} 
Coefficient & Value & exp. (Coefficient) & SE & $\mathbf{z}$ & $\mathbf{p}$ \\
\hline Control $20^{\circ} \mathrm{C}$ & -0.125 & 0.882 & 0.090 & -1.40 & 0.160 \\
Increased warming & -0.220 & 0.803 & 0.090 & -2.43 & 0.015 \\
Slow temp. cycles & -0.070 & 0.932 & 0.089 & -0.79 & 0.430 \\
Warm $25^{\circ} \mathrm{C}$ & -0.217 & 0.805 & 0.090 & -2.42 & 0.016 \\
Temp. treatment: $25^{\circ} \mathrm{C}$ & 1.674 & 5.336 & 0.062 & 26.80 & $<0.001$
\end{tabular}

By simulating environments with different length of temperature cycles we confirmed that faster cycles can select for adaptations that will give shorter lives even when reversible plasticity can evolve. When background mortality is low, we generally find in our simulations that short environmental cycles select for lower reproductive rates and longer lives (Supplementary figure 2), but when background mortality is higher there are two different evolutionary outcomes. Either individuals are highly reversibly plastic and live for a relatively long time or individuals can invest little in reversible plasticity but more in reproduction and thus live shorter lives than individuals in longer environmental cycles (Figure 3). Very similar results were obtained independently of how large fitness effects of mismatching the environment were, so we have only shown results for $m=1$. 
A

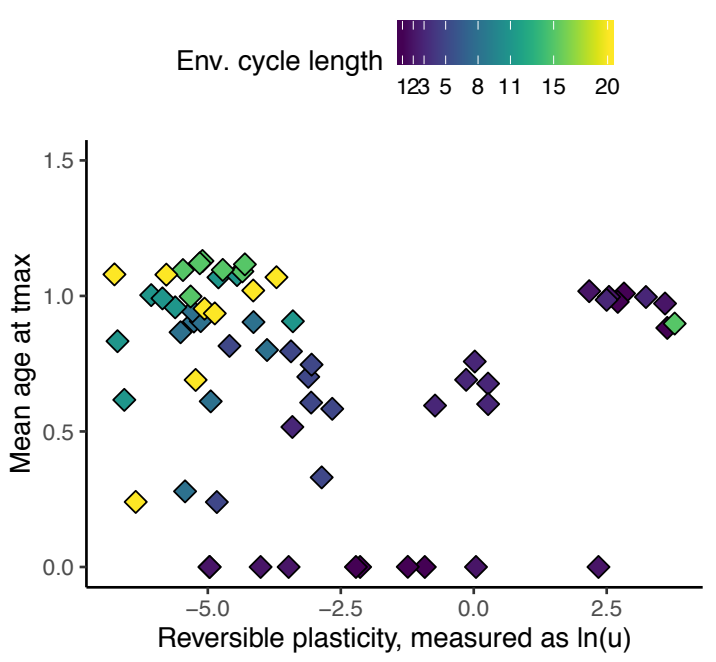

B

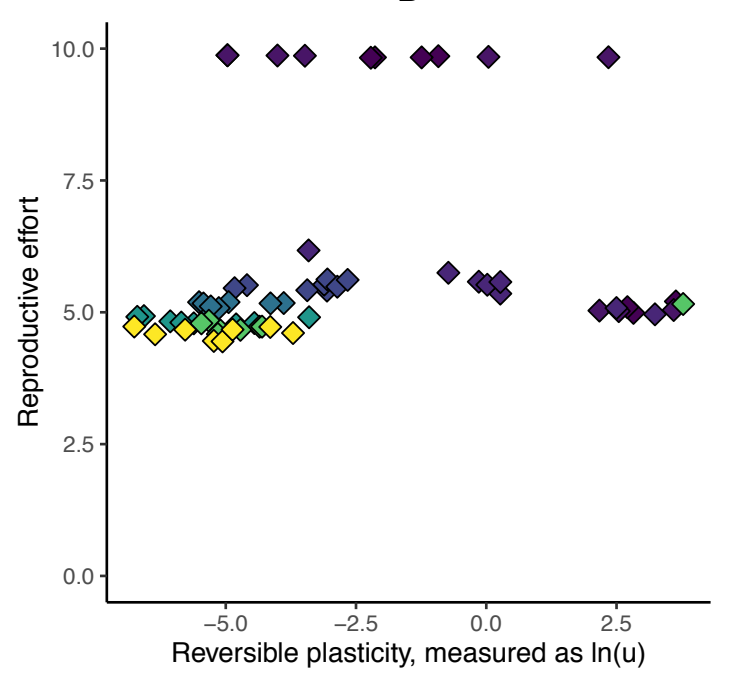

Figure 3. Simulation results for population mean trait values after 20000 time steps with high background mortality $\left(\alpha_{0}=0.25\right)$. When the environmental cycle is intermediate to long (green to yellow diamonds) there is little plasticity (A \& B), reproductive effort is relatively low (B) and lifespans are relatively long. For shorter environmental cycles (blue diamonds) two different evolutionary outcomes are emerging, either individuals invest more in plasticity and keep long lifespan and low reproductive effort, or individuals do not evolve increased plasticity and will have short lives but invest a lot in reproduction.

\section{Discussion}

Environmental heterogeneity is expected to influence the life-history of organisms, but theoretical predictions from different theories range from the evolution of robust, slow-reproducing long-lived organisms to fragile, fast-reproducing short-lived organisms (Murphy 1968; Tuljapurkar and Orzack 1980; Charlesworth 1994; Wilbur and Rudolf 2006; Morris et al. 2008; Cotto and Ronce 2014; Ratikainen and Kokko 2019). Using experimental evolution in the nematode C. remanei, we found that environmental variation influenced the evolution of lifespan in populations with overlapping generations, and that evolution in an environment where the cycles occurred every second generation resulted in the evolution of short lifespan. This was 
also confirmed by simulating evolution of plasticity and lifespan in environments with environmental cycles of different length showing the possibility of evolution of both short and long lifespan in fast fluctuations.

The traditional view has long been that environmental heterogeneity should result in the evolution of increased somatic maintenance, since it would enable the organism to survive unfavourable periods in order to reproduce under more favourable conditions (Murphy 1968; Tuljapurkar and Orzack 1980; Charlesworth 1994; Wilbur and Rudolf 2006; Morris et al. 2008). The effect is, however, expected to be small (Benton and Grant 1999) and the theory has limited empirical support (reviewed in (Roff 2002). Instead, alternative theories have been proposed, which suggest that the strategy to deal with the phenotypic mismatch occurring when the environment changes is central (Cotto and Ronce 2014; Ratikainen and Kokko 2019). If the organism is able to adjust its phenotype to avoid mismatch, reversible plasticity and long lifespan is expected to coevolve in variable environments (Ratikainen and Kokko 2019). However, if reversible plasticity is too costly or not able to evolve, the increased likelihood of an environmental change with increasing age would select for increased investment into early-life fitness resulting in the evolution of short lifespan (Cotto and Ronce 2014; Ratikainen and Kokko 2019). This is based upon the observation that adaptation is never instantaneous; instead, an adapting population has not yet reached the new fitness peak (Lande and Shannon 1996; Kopp and Matuszewski 2014). Since selection is weaker for traits expressed in late-life (Medawar 1952; Williams 1966; Caswell and Shyu 2017) older individuals will be further from the new fitness peak. If the population is age-structured, this means that an adapting population has reduced fitness, and fitness is most reduced for old individuals, resulting in weaker selection 
and therefore increased accumulation of mutations whose effects on fitness are concentrated in late-life (Cotto and Ronce 2014), following the logic of the mutation accumulation theory of ageing (Medawar 1952; Charlesworth 1994). This effect is expected to be most accentuated when the environment fluctuates with a period just longer than the generation time, since old individuals are more likely to end up in the environment they did not develop in and thus not are adapted to.

We found that the lines in Fast temperature cycles had evolved a shorter lifespan than the Warm $25^{\circ} \mathrm{C}$ and Increased warming lines in both testing temperatures. This is important because the Fast temperature cycles and Increased warming lines had experienced the same average temperature over evolutionary time. This supports theoretical predictions in cases where reversible plasticity does not evolve (Ratikainen and Kokko 2019). Phenotypic plasticity is common in nematodes (Viney and Diaz 2012), but most examples concern irreversible developmental plasticity. Since cell division of somatic cells stops at sexual maturity in Caenorhabditis nematodes, there are limited possibilities for major morphological changes after maturation, except for growth of cell size. On the other hand, nematodes have genetic variation for behavioural traits, such as locomotion, dispersal and learning (Ardiel and Rankin 2010; Chen and Maklakov 2014; Zwoinska et al. 2016), which classify as reversible plasticity, and the evolution of such plasticity and the co-evolution of longer lifespan is an alternative evolutionary outcome in our simulations.

While our results qualitatively support predictions from recent theory (Cotto and Ronce 2014; Ratikainen and Kokko 2019), we are not certain that the underlying mechanism is the same as proposed by (Cotto and Ronce 2014). While they predict 
that a shorter lifespan should evolve via increased mutation accumulation, we suggest this a less likely factor to have such a large effect on the outcome of this experiment over only 30 generations of experimental evolution. Instead, our extension of the Ratikainen and Kokko model (Ratikainen and Kokko 2019) suggest that this could be caused by selection for early-life fitness because old individuals will often end up in the "wrong" environment.

While maladaptation is predicted to result in the evolution of short lifespan also in novel but stable environments (Cotto and Ronce 2014), we found instead the evolution of long lifespan in both warm and slow increase in warming treatments. The discrepancy between theory and our results is likely a consequence of the model being very general and focusing on the maladaptation and mismatch occurring during adaptation to a new environment, but is not considering that the environment itself may select for a certain life-history strategy. In this experiment, the novel environment was both warm and stressful, and heat-shock resistance is associated with long life in nematodes (Amrit et al. 2010; Chen and Maklakov 2012; Lind et al. 2017). In general, heat-shock proteins protect the organisms against a broad range of stresses (Sørensen et al. 2003) and there is a link between long life and heat shock resistance across taxa (Rose et al. 1992; Holzenberger et al. 2003). Thus, while maladaptation in the novel warm but quite stable environment may select for short lifespan (Cotto and Ronce 2014), it is likely swamped by strong selection for heatshock resistance resulting in the evolution of longer lifespan.

In conclusion, we found that the presence of environmental variation is a key driver of the evolution of lifespan. Specifically, fast temperature fluctuations where the 
organism is likely to experience a switch to a different environment in late-life select for short lifespan, while stable but stressful warm environments select for long lifespan. Since ongoing climate warming results in both warmer and more variable temperatures across the planet (Vasseur et al. 2014; Vázquez et al. 2017), understanding the effect of temperature fluctuations in relation to the generation time of the organisms is vital for predicting the evolution of life-histories.

\section{Acknowledgement}

This work was funded by the Swedish Research Council [grant number 2016-05195 to MIL, grant number 621-2013-4828 to AAM], the European Research Council [grants AGINGSEXDIFF and GERMLINEAGEINGSOMA to AAM] and IIR was supported by the Norwegian Research Council [grant number 240008 and 223257]. We thank Frank Johansson, Anssi Laurila, and Claus Rüffler for discussions.

\section{Author contributions}

MIL and AAM designed the experiment, MIL, JA, HC, TK and TL performed experimental evolution, MIL and JA performed phenotypic assays, MIL analysed the data, IIR performed the simulation. MIL drafted the manuscript together with AAM and IIR. All authors contributed to the revision of the manuscript. 


\section{References}

Amrit, F. R. G., C. M. L. Boehnisch, and R. C. May. 2010. Phenotypic covariance of longevity, immunity and stress resistance in the Caenorhabditis nematodes. PLoS ONE 5:e9978.

Ardiel, E. L., and C. H. Rankin. 2010. An elegant mind: Learning and memory in Caenorhabditis elegans. Learn. Mem. 17:191-201.

Benton, T. G., and A. Grant. 1999. Optimal reproductive effort in stochastic, densitydependent environments. Evolution 53:677-688.

Botero, C. A., F. J. Weissing, J. Wright, and D. R. Rubenstein. 2015. Evolutionary tipping points in the capacity to adapt to environmental change. Proc. Natl. Acad. Sci. 112:184-189.

Bubliy, O. A., and V. Loeschcke. 2005. Correlated responses to selection for stress resistance and longevity in a laboratory population of Drosophila melanogaster. J. Evol. Biol. 18:789-803.

Caswell, H., and E. Shyu. 2017. Senescence, selection gradients and mortality. Pp. 56-82 in R. P. Shefferson, O. R. Jones, and R. Salguero-Gómez, eds. The evolution of senescence in the tree of life. Cambridge University Press, Cambridge, UK.

Charlesworth, B. 1994. Evolution in age-structured populations. 2nd ed. Cambridge University Press, New York, NY, USA.

Chen, H., and A. A. Maklakov. 2014. Condition dependence of male mortality drives the evolution of sex differences in longevity. Curr. Biol. 24:2423-2427. 
Chen, H., and A. A. Maklakov. 2012. Longer life span evolves under high rates of condition-dependent mortality. Curr. Biol. 22:2140-2143.

Chen, H. -y., F. Spagopoulou, and A. A. Maklakov. 2016. Evolution of male agespecific reproduction under differential risks and causes of death: males pay the cost of high female fitness. J. Evol. Biol. 29:848-856.

Cotto, O., and O. Ronce. 2014. Maladaptation as a source of senescence in habitats variable in space and time. Evolution 68:2481-2493.

Hamilton, W. D. 1966. The moulding of senescence by natural selection. J. Theor. Biol. 12:12-45.

Holzenberger, M., J. Dupont, B. Ducos, P. Leneuve, A. Géloën, P. C. Even, P. Cervera, and Y. Le Bouc. 2003. IGF-1 receptor regulates lifespan and resistance to oxidative stress in mice. Nature 421:182-187.

Johansson, F., and L. Rowe. 1999. Life history and behavioral responses to time constraints in a damselfly. Ecology 80:1242-1252.

Kenyon, C. 2005. The plasticity of aging: insights from long-lived mutants. Cell 120:449-460.

Kopp, M., and S. Matuszewski. 2014. Rapid evolution of quantitative traits: theoretical perspectives. Evol. Appl. 7:169-191.

Lande, R., and S. Shannon. 1996. The role of genetic variation in adaptation and population persistence in a changing environment. Evolution 50:434-437. 
Laugen, A. T., A. Laurila, K. Räsänen, and J. Merilä. 2003. Latitudinal

countergradient variation in the common frog (Rana temporaria) development rates evidence for local adaptation. J. Evol. Biol. 16:996-1005.

Lee, W.-S., P. Monaghan, and N. B. Metcalfe. 2013. Experimental demonstration of the growth rate-lifespan trade-off. Proc. R. Soc. B Biol. Sci. 280:20122370.

Lind, M. I., H. Chen, S. Meurling, A. C. Guevara Gil, H. Carlsson, M. K. Zwoinska, J. Andersson, T. Larva, and A. A. Maklakov. 2017. Slow development as an evolutionary cost of long life. Funct. Ecol. 31:1252-1261.

Lind, M. I., P. K. Ingvarsson, H. Johansson, D. Hall, and F. Johansson. 2011. Gene flow and selection on phenotypic plasticity in an island system of Rana temporaria. Evolution 65:684-697.

Lind, M. I., M. K. Zwoinska, J. Andersson, H. Carlsson, T. Krieg, T. Larva, and A. A. Maklakov. 2019. Environmental variation mediates the evolution of anticipatory parental effects. bioRxiv, doi: 10.1101/606103.

Lind, M. I., M. K. Zwoinska, S. Meurling, H. Carlsson, and A. A. Maklakov. 2016. Sex-specific trade-offs with growth and fitness following lifespan extension by rapamycin in an outcrossing nematode, Caenorhabditis remanei. J. Gerontol. A. Biol. Sci. Med. Sci. 71:882-890.

Lionaki, E., and N. Tavernarakis. 2013. Assessing aging and senescent decline in Caenorhabditis elegans: cohort survival analysis. Pp. 473-484 in L. Galluzzi, I. Vitale, O. Kepp, and G. Kroemer, eds. Cell Senescence. Humana Press, New York. Medawar, P. B. 1952. An unresolved problem in biology. Lewis, London, UK. 
Morris, W. F., C. A. Pfister, S. Tuljapurkar, C. V. Haridas, C. L. Boggs, M. S. Boyce,

E. M. Bruna, D. R. Church, T. Coulson, D. F. Doak, S. Forsyth, J.-M. Gaillard, C. C.

Horvitz, S. Kalisz, B. E. Kendall, T. M. Knight, C. T. Lee, and E. S. Menges. 2008.

Longevity can buffer plant and animal populations against changing climatic

variability. Ecology 89:19-25.

Murphy, G. I. 1968. Pattern in life history and the environment. Am. Nat. 102:391403.

Parsons, P. A. 1995. Inherited stress resistance and longevity: a stress theory of ageing. Heredity 75:216-221.

Ratikainen, I. I., and H. Kokko. 2019. The coevolution of lifespan and reversible plasticity. Nat. Commun. 10:538.

Reznick, D. N., M. J. Bryant, D. Roff, C. K. Ghalambor, and D. E. Ghalambor. 2004. Effect of extrinsic mortality on the evolution of senescence in guppies. Nature 431:1095-1099.

Roff, D. A. 2002. Life history evolution. Sinauer Associates Inc, Sunderland, USA.

Rose, M. R., L. N. Vu, S. U. Park, and J. L. Graves. 1992. Selection on stress resistance increases longevity in Drosophila melanogaster. Exp. Gerontol. 27:241250.

Sørensen, J. G., T. N. Kristensen, and V. Loeschcke. 2003. The evolutionary and ecological role of heat shock proteins. Ecol. Lett. 6:1025-1037.

Stearns, S. C. 1992. The evolution of life histories. Oxford University Press, New York, NY, USA. 
Stearns, S. C., M. Ackermann, M. Doebeli, and M. Kaiser. 2000. Experimental evolution of aging, growth, and reproduction in fruitflies. Proc. Natl. Acad. Sci. 97:3309-3313.

Stiernagle, T. 2006. Maintenance of C. elegans. WormBook Online Rev. C Elegans Biol., doi: 10.1895/wormbook.1.101.1.

Stocker, T. F. 2014. Climate change 2013: the physical science basis: Working Group I contribution to the Fifth assessment report of the Intergovernmental Panel on Climate Change. Cambridge University Press.

Tufto, J. 2015. Genetic evolution, plasticity, and bet-hedging as adaptive responses to temporally autocorrelated fluctuating selection: A quantitative genetic model. Evolution 69:2034-2049.

Tuljapurkar, S. D., and S. H. Orzack. 1980. Population dynamics in variable environments I. Long-run growth rates and extinction. Theor. Popul. Biol. 18:314342.

Vasseur, D. A., J. P. DeLong, B. Gilbert, H. S. Greig, C. D. G. Harley, K. S. McCann, V. Savage, T. D. Tunney, and M. I. O’Connor. 2014. Increased temperature variation poses a greater risk to species than climate warming. Proc. R. Soc. B Biol. Sci. 281:20132612.

Vázquez, D. P., E. Gianoli, W. F. Morris, and F. Bozinovic. 2017. Ecological and evolutionary impacts of changing climatic variability. Biol. Rev. 92:22-42.

Viney, M., and A. Diaz. 2012. Phenotypic plasticity in nematodes: Evolutionary and ecological significance. Worm 1:98-106. 
Wilbur, H. M., and V. H. W. Rudolf. 2006. Life-history evolution in uncertain environments: bet hedging in time. Am. Nat. 168:398-411.

Williams, G. C. 1966. Adaptation and natural selection. Princeton University Press, Princeton, N.J.

Williams, P. D., T. Day, Q. Fletcher, and L. Rowe. 2006. The shaping of senescence in the wild. Trends Ecol. Evol. 21:458-463.

Zwoinska, M. K., M. I. Lind, M. Cortazar-Chinarro, M. Ramsden, and A. A.

Maklakov. 2016. Selection on learning performance results in the correlated evolution of sexual dimorphism in life-history. Evolution 70:342-357. 